\title{
烯丙醇在亲核取代反应中的研究进展
}

\author{
张小祥 $*, a$ 孙小萍 ${ }^{a}$ 谈继淮 ${ }^{a}$ 樊 辉 ${ }^{b}$ 饶卫东*, $a$ \\ ( ${ }^{a}$ 南京林业大学化工学院 江苏省生物质绿色燃料与化学品重点实验室 南京 210037)

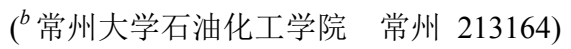

\begin{abstract}
摘要 烯丙醇的亲核取代反应在有机合成化学中具有十分重要的地位, 该反应可被广泛应用于具有生物活性的药物以 及天然产物的合成. 烯丙基化反应也是一类十分重要的引入 C-3 结构单元的合成方法学. 这种合成方法的优点在于双 键的保留, 使得分子的官能团可以进行进一步转化. 传统的 Tsuji-Trost 烯丙基化方法会产生大量的废弃物, 相比之下 直接以烯丙醇烯丙基化方法是一种绿色的合成方法. 综述了烯丙醇在路易斯酸或者布朗斯特酸催化下与不同的亲核试 剂发生分子内和分子间亲核取代反应，构筑 $\mathrm{C}-\mathrm{X}(\mathrm{X}=\mathrm{C}, \mathrm{N}, \mathrm{O}, \mathrm{S})$ 键的最新研究进展，涉及到芳基化合物、羰基化合物、 氨基或者磺胺类化合物以及醇类等不同种类亲核试剂. 最后就烯丙醇烯丙基化研究及应用中存在的问题和难点对其前 景进行了展望.
\end{abstract}

关键词 烯丙醇; 亲核取代; 均相催化; 烯丙基化

\section{Progress of Nucleophilic Substitution of Allylic Alcohols}

\author{
Zhang, Xiaoxiang ${ }^{*, a} \quad$ Sun, Xiaoping ${ }^{a} \quad{\text { Tan, } \text { Jihuai }^{a} \quad \text { Fan, Hui }}^{b} \quad$ Rao, Weidong ${ }^{*, a}$ \\ ( ${ }^{a}$ Jiangsu Key Laboratory of Biomass-based Green Fuels and Chemicals, College of Chemical Engineering, Nanjing Forestry \\ University, Nanjing 210037) \\ ( ${ }^{b}$ School of Petrochemical Engineering, Changzhou University, Changzhou 213164)
}

\begin{abstract}
Nucleophilic substitution of allylic alcohols is very important in organic synthetic chemistry, which could be used for the synthesis of bioactive pharmaceutic compounds and natural compounds. One of the most powerful and efficient methods to introduce a C-3 unit is the allylic reaction. The advantage of this synthetic approach is the retention of the $\mathrm{C}=\mathrm{C}$ bond in the product that can act as a handle for further functional group transformations. One synthetic strategy that has been often relied upon to introduce this functional group is the Tsuji-Trost reaction. However, a drawback of this method is the generation of waste products resulting from displacement of the leaving group on treating with a catalyst and/or nucleophile. Therefore, the direct allylation of allylic alcohols is considered as a green method. In this review, the latest research progress on the Lewis and Brønsted acids catalyzed intermolecular and intramolecular nucleophilic substitution for the formation of $\mathrm{C}-\mathrm{X}(\mathrm{X}=\mathrm{C}, \mathrm{N}, \mathrm{O}, \mathrm{S})$ bond is presented, and a variety of nucleophiles, such as aromatical compounds, carbonyl compounds, amines or sulfonamides, alcohols and so on, are discussed. Finally, the problems and difficulties in research and application of allylation are discussed and then prospective is provided.
\end{abstract}

Keywords allylic alcohols; nucleophilic substitution; homogeneus catalyzed; allylation

烯丙基化反应是有机化学中构筑碳碳、碳杂键的一 类十分重要的反应, 其烯丙基化产物在天然产物以及具 有生物活性化合物的合成中应用十分广泛 ${ }^{[1]}$. 有机合成 中引入 C-3 单元最为简单有效的方法之一是通过烯丙基 化反应, 这种合成方法的特点在于 $\mathrm{C}=\mathrm{C}$ 双键的保留, 可以进行官能团进一步转化反应, 从而形成复杂结构分
子 $^{[2]}$. 正因为烯丙基化是如此重要, 几十年来, 人们进 行了各种各样相关的合成方法研究, 大量的研究成果被 报道出来 ${ }^{[3]}$. 传统上, 我们可以将烯丙基化反应归纳为 两大类反应，一大类是利用过量的碱和烯丙基卤化物来 完成烯丙基化; 另一大类是所谓的 Tsuji-Trost 反应, 即 利用过渡金属催化活化的烯丙醇衍生物(如烯丙基卤化

*E-mail: s070038@hotmail.com; wdrao15@163.com

Received May 5, 2015; revised June 5, 2015; published online June 19, 2015.

Project supported by the Young National Natural Science Foundation of China Grant (No. 21302096), the Young Natural Science Foundation of Jiangsu Province (No. BK20130962) and the Project Fund from the Priority Academic Program Development of Jiangsu Higher, Education Institutions (PAPD).

国家自然科学青年基金(No. 21302096 )、江苏省自然科学青年基金(No. BK20130962)、江苏高校优势学科建设工程(PAPD)资助项目. 
物，烯丙基酯等)，经过一个 $\pi$-金属烯丙基中间体来完成 烯丙基化. 这两类方法都有其严重的缺陷, 在活性原料 的制备以及反应的进行中将会产生大量的卤化氢、羧酸 盐、碳酸盐、三氟甲磺酸或者磷酸盐等废弃物 ${ }^{[4]}$, 这些 酸性废弃物具有强酸性或者腐蚀性, 对环境的污染较 大. 另外随着人们对绿色化学的重视, 传统上的两大类 烯丙基化构建方法不再具有优势, 发展环境友好的绿色 合成方法变得尤为重要. 近年来, 人们发现醇羟基邻位 的 $\pi$ 键具有致活功能, 能够在反应中起到稳定碳正离子 的作用, 从而促进了反应的进一步进行 ${ }^{[4]}$.

因此, 烯丙醇本质上是一种活性醇化合物, 只要在 合适催化剂的作用下, 可以作为亲电试剂的前驱体, 直 接与亲核试剂作用生成烯丙基化产物, 而且生成的副产 物只有水, 不产生污染环境的废弃物, 很显然这是一种 绿色的烯丙基化合成方法(Scheme 1). 目前, 环境友好 型的烯丙醇作为一种替代传统烯丙基化试剂正在逐步 得到大家的重视, 近年来在该领域发表了大量的相关文 章 $^{[5,6]}$.

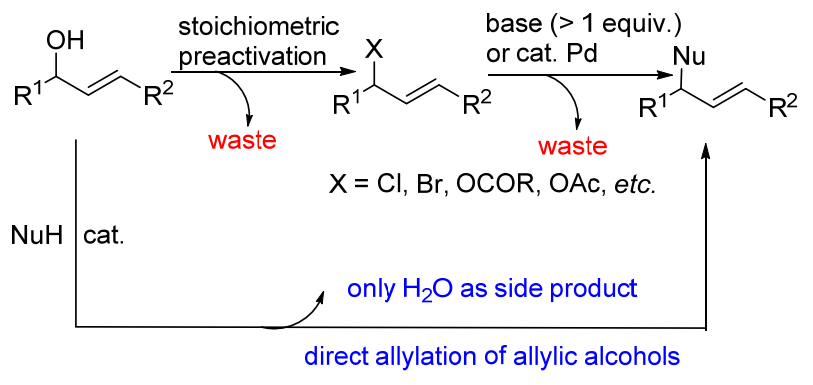

图式 1 烯丙醇的烯丙基化反应

Scheme 1 Allylation of allylic alcohols

本文结合本人最近以及博士期间在该领域的研究 工作综述近年来国内外研究人员直接利用烯丙醇进行 烯丙基化反应的研究进展, 并针对研究中存在的问题对 其前景进行展望.

\section{1 烯丙醇在亲核取代反应中的应用}

在有机合成中, 烯丙基化方法于 1965 年首次体现 在 Tsuji-Trost 反应中(Eq. 1) ${ }^{[7]}$. 这种烯丙基烷基化合成 方法的缺点是会产生大量酸性废弃物, 对环境的污染较 大. 为了减轻对环境污染的压力, 近年来对此方法作了 很多的改进, 发表了大量的以烯丙醇直接烯丙基化的文 章.

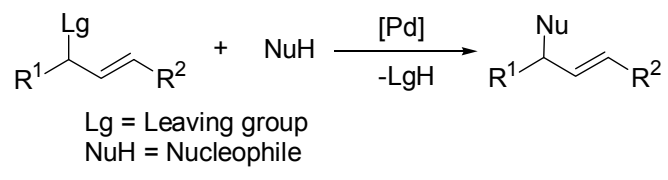

\section{1 烯丙醇与芳基衍生物分子间烯丙基化反应}

早在 1997 年, Fukuzawa 等 ${ }^{[8]}$ 以烯丙醇和苯为原料, 在 $\mathrm{Sc}(\mathrm{OTf})_{3}$ 催化作用下进行傅克烷基化反应(表 1, Entry 1 ), 得到 48\% 95\% 烯丙基苯衍生物, 只有少量的副产 物产生. 在该反应中苯作为亲核试剂进攻取代基较少的 烯丙基碳, 缺点在于该反应只适用于少数几种类型的脂 肪族烯丙醇与苯的反应，当使用环状烯丙醇时，烯丙基 化反应就不能发生. 随后, 人们还发现了很多其它 Lewis 和 Brønsted 酸对以烯丙醇为原料的烯丙基化反应 有着良好的催化作用.

2008 年, $\mathrm{Liu}$ 等 ${ }^{[9]}$ 报道了以 $\mathrm{AlCl}_{3}$ 催化剂, 催化硫醇 保护的 $\alpha$-羟基烯酮与各种芳烃的烯丙基化反应 (表 1 , Entry 2). 研究表明, 与 $\mathrm{C}=\mathrm{C}$ 双键相连的含有吸电子基 团的 Morita-Baylis-Hillman (MBH)醇可以认为是一种特 殊的烯丙醇, 因为它们可以在烯丙基化反应后，进一步 发生分子内环合反应, 生成一系列具有良好的生物活性 和药物性能的重要化合物, 例如二氢香兰素衍生物等.

2009 年, Chan 课题组 ${ }^{[10]}$ 也发表了 $\mathrm{MBH}$ 醇被 $\mathrm{FeCl}_{3} \cdot 6 \mathrm{H}_{2} \mathrm{O}$ 催化的直接 $\alpha$-位亲核取代反应(Eq. 2), 亲核 试剂不仅可以用芳基类(如苯酚、硫酚), 还可以用醇以 及硫醇类化合物. 另外 Chan 等还通过合成手性 MBH 醇 原料与亲核试剂反应，得到消旋产物，间接验证了该反 应是一个涉及碳正离子的过程. 近年来, 金络合物作为 烯丙基化反应的有效催化剂, 已经受到越来越多的关 注. 2008 年, Chan 课题组 ${ }^{[11]}$ 以芳烃或杂环芳烃和烯丙醇 为原料, $\mathrm{AuCl}_{3}$ 为催化剂, 进行烯丙基烷基化反应(表 1 , Entry 3). 研究结果表明, $\mathrm{AuCl}_{3}$ 的催化性能优异, 在常 温条件下，相应产物收率高达 $99 \%$ ，且产物具有良好的 区域选择性. 1 年以后, $\mathrm{Liu}$ 等 ${ }^{[12]}$ 提出了一种由烯炔醇和 呋喃在 Gagosz 催化剂 $\left(\mathrm{Ph}_{3} \mathrm{P}\right) \mathrm{AuNTf}_{2}$ 作用下进行串联反 应制备芳基烯酮的通用方法. 研究结果表明，该金配合 物催化剂在该串联反应的两个反应部分都是高效催化 剂, 相应产物的收率高达 91\%(表 1, Entry 4).

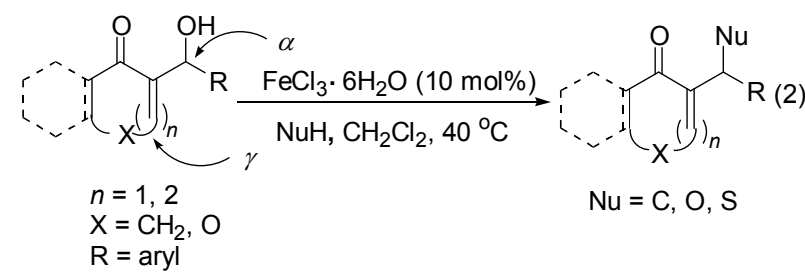

2010 年, McCubbin 等 ${ }^{[13]}$ 报道了烯丙醇和富电子的 芳烃以及杂环芳烃在 $\mathrm{C}_{6} \mathrm{~F}_{5} \mathrm{~B}(\mathrm{OH})_{2}$ 催化作用下, 进行烯 丙基化反应(表 1, Entry 5). 当催化剂用量为 $10 \mathrm{~mol} \%$ 时, 目标产物的产率可达 $99 \%$. 该合成工艺的优点在于催化 剂 $\mathrm{C}_{6} \mathrm{~F}_{5} \mathrm{~B}(\mathrm{OH})_{2}$ 能有效地与空间位阻较大的醇络合, 另 外硼系列催化剂能溶于大多数有机溶剂, 且对水和空气 
表 1 酸催化分子间烯丙基化反应

Table 1 Acid catalyzed intermolecular allylation

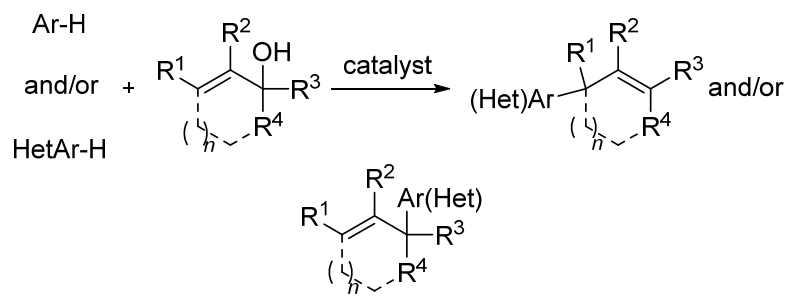

\begin{tabular}{|c|c|c|c|}
\hline Entry & Catalyst & Yield/\% & Ref. \\
\hline 1 & $\mathrm{Sc}(\mathrm{OTf})_{3}$ & $48 \sim 95$ & [8] \\
\hline 2 & $\mathrm{AlCl}_{3}$ & $50 \sim 90$ & [9] \\
\hline 3 & $\mathrm{AuCl}_{3}$ & $50 \sim 99$ & [11] \\
\hline 4 & $\left(\mathrm{Ph}_{3} \mathrm{P}\right) \mathrm{AuNTf}_{2}$ & $33 \sim 91$ & [12] \\
\hline 5 & $\mathrm{C}_{6} \mathrm{~F}_{5} \mathrm{~B}(\mathrm{OH})_{2}$ & $0 \sim 99$ & [13] \\
\hline 6 & $\mathrm{Ca}\left(\mathrm{NTf}_{2}\right)_{2}$ & $0 \sim 88$ & [14] \\
\hline 7 & $p-\mathrm{TsOH} \cdot \mathrm{H}_{2} \mathrm{O}$ & $55 \sim 84$ & [16] \\
\hline 8 & $\mathrm{TfOH}$ & $55 \sim 95$ & [17] \\
\hline 9 & Calix $[n]$ arene sulfonic acid & $60 \sim 94$ & [18] \\
\hline
\end{tabular}

相对稳定，而且可通过简单萃取的方法，十分容易地从 复杂混合物中将硼催化剂进行回收利用.

同年, Niggemann 等 ${ }^{[14]}$ 报道了 $\mathrm{Ca}\left(\mathrm{NTf}_{2}\right)_{2}$ 在室温条件 下, 催化富电子芳烃和烯丙醇亲核取代反应, 相应的产 物产率可达 $88 \%$ (表 1, Entry 6). 研究结果表明, 该反应 只有在以 $\mathrm{Bu}_{4} \mathrm{NPF}_{6}$ 为助剂的条件下, 才能进行烯丙基化 反应. 值得注意的是, 该催化体系可以催化羟基的 位置没有活化基团的烯丙醇, 这是在用其它催化剂催化 类似反应中十分罕见的 ${ }^{[15]}$.

除了 Lewis 酸之外, Brønsted 酸也是烯丙基化反应 中十分重要的催化剂. 2006 年, Sanz 等 ${ }^{[16]}$ 使用布朗斯特 酸 $p-\mathrm{TsOH} \cdot \mathrm{H}_{2} \mathrm{O}$ 为催化剂, 催化芳烃和吲哚类衍生物直 接烯丙基化反应, 产物具有较高的选择性和产率(表 1 , Entry 7). 反应是在敞口瓶中分析纯溶剂下进行的, 无惰 性气体保护, 不仅对环境友好, 而且催化剂成本也低, 使得该反应的大规模合成成为可能. 1 年之后, Bras 等 ${ }^{[17]}$ 拓展了该合成方法, 通过 TfOH 催化富电子芳烃的方法, 在无溶剂的条件下，相应产物的产率高达 95\%(表 1, Entry 8).

大多数烯丙基化反应是在有机溶剂中进行的, 然而 有人尝试了用水作为溶剂, 发现该类反应也是可行的, 这对于发展绿色化学合成方法是一个十分重要的贡献. 2008 年, Wang 等 ${ }^{[18]}$ 以具有表面活性剂性质的 calix $[n]$ arene 磺酸为固体酸催化剂, 在水中催化芳烃和 杂环芳烃进行烯丙基化反应(表 1, Entry 9). 研究结果表 明, 该催化剂同样可以获得相应的烯丙基化产物, 并且 产物收率高达 $94 \%$. 除了能够在水中使用之外, 该催化 剂的突出优点还在于可以回收重复使用, 而且经实验在 重复使用 7 次之后, 该催化剂的催化活性并没有显著降
低.

2006 年, $\mathrm{Baba}$ 等 ${ }^{[19]}$ 报道了吲哚和烯丙醇在 $\mathrm{InCl}_{3}$ 催 化作用下直接烯丙基化反应的研究(表 2, Entry 1). 结果 表明, 两种吲哚 C-3 位烯丙基化产物的产率为 64\% $78 \%$ ，且产物具有较高的区域选择性，没有检测到 C-2 或者 $\mathrm{N}$ 上烷基化产物. 然而, 该反应的缺点在于该方法 没有进行不同种类的底物的拓展，只做了两个底物的反 应，存在应用普适性测试的局限性. 随后，Yadav 等 ${ }^{[20]}$ 对上述合成方法进行了大量的底物拓展研究(表 2, Entry 2). 研究发现, 当选用 $\operatorname{InBr}_{3}$ 作为催化剂时, 常规的吲哚 衍生物和烯丙醇进行烷基化反应能够取得较好的产率. 与此同时, Jana 等 ${ }^{[21]}$ 发现了 $\mathrm{FeCl}_{3}$ 对吲哚 C-3 位置上的烯 丙基烷基化反应同样具有良好的催化活性和高的选择 性，相应的产物收率 56\% 98\%(表 2, Entry 3).

表 2 Lewis 酸催化吲哚的烯丙基化反应

Table 2 Lewis acid catalyzed allylation of indoles

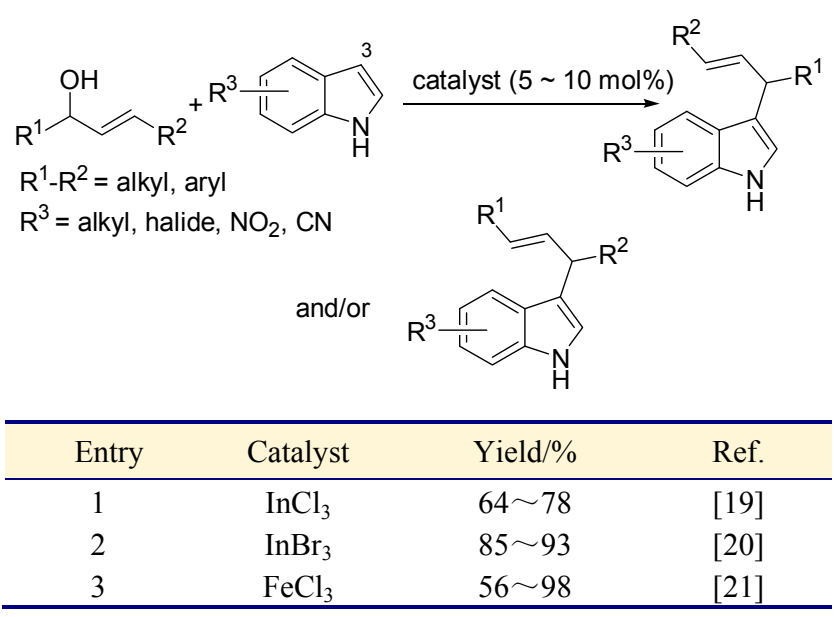

\section{2 丙醇与芳基衍生物分子内烯丙基化反应}

$\mathrm{Li}$ 等 ${ }^{[22]}$ 报道了烯丙醇分子内烷基化反应作为有效 方法合成狮衍生物(表 3, Entry 1). 以三氟化硼乙醚 $\left(\mathrm{BF}_{3} \cdot \mathrm{Et}_{2} \mathrm{O}\right)$ 作为催化剂, 在温和条件下, 催化芳基烯丙 醇进行分子内烷基化反应，得到相应的产物碘代狮衍生 物 $\left(\mathrm{R}^{2}=\mathrm{I}\right)$ 的产率为 $55 \% \sim 90 \%$, 经该反应获得的碘代产 物可以通过 Suzuki 偶联反应构筑多芳基狮衍生物. 同 年, $\mathrm{Liu}$ 等 ${ }^{[23]}$ 报道了 $\mathrm{TsOH} \cdot \mathrm{H}_{2} \mathrm{O}$ 催化芳基烯丙醇分子内 烷基化反应, 生成了具有高取代和螺环结构的狮衍生物 (表 3, Entry 2). 随后 Zhou 等 ${ }^{[24]}$ 报道了在 $\mathrm{FeCl}_{3} \cdot 6 \mathrm{H}_{2} \mathrm{O}$ 催 化剂作用下，同样可以生成多取代狮取代衍生物，收率 高达 93\%(表 3, Entry 3). 一个显著的特点是该反应所使 用的金属催化剂是催化量的，而前面两个反应非金属催 化剂则是需要等物质的量.

烯丙醇除了可以进行分子内环内反应，还可以进行 分子内环外反应. 2008 年, Nishizawa 等 ${ }^{[25]}$ 报道了以烯丙 醇为起始原料, 在 $\mathrm{Hg}(\mathrm{OTf})_{2}$ 的催化作用下, 烯丙醇发生 
表 3 酸催化分子内傅克烷基化反应

Table 3 Acid catalyzed intramolecular Friedel-Crafts allylic alkylation

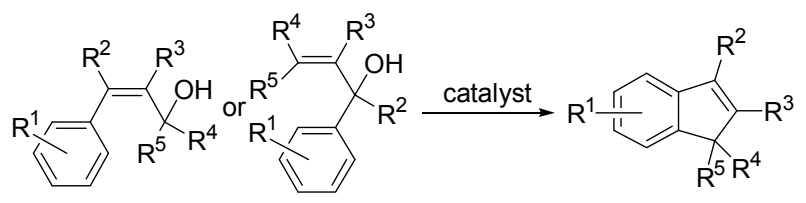

$$
\begin{aligned}
& R^{1}=H, \text { alkyl, halide } \\
& R^{2}=H, \text { alkyl, aryl, halide } \\
& R^{3}-R^{5}=H, \text { alkyl aryl }
\end{aligned}
$$

\begin{tabular}{cccc}
\hline Entry & Catalyst & Yield/\% & Ref. \\
\hline 1 & $\mathrm{BF}_{3} \bullet \mathrm{Et}_{2} \mathrm{O}$ & $55 \sim 90$ & {$[22]$} \\
2 & $\mathrm{TsOH} \cdot \mathrm{H}_{2} \mathrm{O}$ & $80 \sim 97$ & {$[23]$} \\
3 & $\mathrm{FeCl}_{3} \cdot 6 \mathrm{H}_{2} \mathrm{O}$ & $56 \sim 93$ & {$[24]$} \\
\hline
\end{tabular}

分子内烷基化反应，生成苯并六元环产物(表 4, Entry 1). 首先是烯丙醇化合物经过傅克环化反应生成有机录中 间体，随后在 $\mathrm{TfOH}$ 活化作用下生成新的中间体，最后 新形成的中间体脱录得到苯并六元环产物. 虽然该合成 方法产率高达 99\%, 但是由于永盐剧毒, 容易导致环境 的污染, 不能算是一种好的合成方法. 一年以后, Bandini 等 ${ }^{[26]}$ 开发了 AgOTf 来替代有毒的丞盐催化剂(表 4, Entry 2), 并进一步拓展了底物, 研究结果表明, 银系列 Lewis 催化剂的催化机理和采系列催化机理一致, 但银 系列催化剂不仅毒性低而且催化剂用量相对较少, 而相 应的产物产率仍高达 90\%。

表 4 Lewis 酸催化烯丙醇分子内傅克六元环化反应

Table 4 Lewis acid catalyzed intramolecular Friedel-Crafts 6-exo-trig cyclization of allylic alcohols
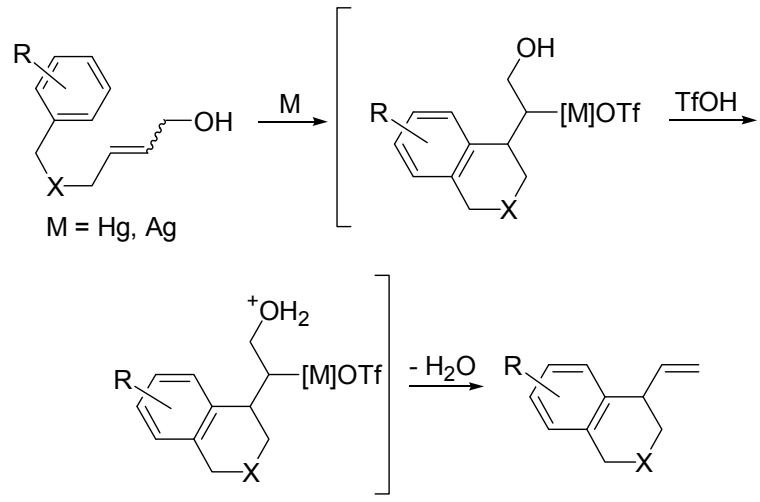

\begin{tabular}{cllll}
\hline Entry & \multicolumn{1}{c}{$[\mathrm{M}]$} & \multicolumn{1}{c}{$\mathrm{X}$} & Yield/\% & Ref. \\
\hline 1 & $\mathrm{Hg}(\mathrm{OTf})_{2}$ & $\mathrm{CH}_{2}$ & $30 \sim 99$ & {$[25]$} \\
2 & $\mathrm{AgOTf}$ & $\mathrm{CH}_{2}$, NHTs, C(COOEt $)_{2}$ & $53 \sim 90$ & {$[26]$} \\
\hline
\end{tabular}

2009 年, Bandini 等 ${ }^{[27]}$ 报道了吲哚一烯丙醇进行分子 内不对称傅克烷基化反应如 Eq. 3 所示. 研究结果表明, 当使用 $\mathrm{AuCl} \cdot \mathrm{Me}_{2} \mathrm{~S}$ 和手性膦配体作为手性催化剂时, 吲 哚并六元环衍生物产率为 $52 \% \sim 87 \%$, 且对映体过量值

(ee 值)高达 $96 \%$. 这是烯丙醇首次直接应用在傅克烯丙 基烷基化不对称催化反应中.<smiles></smiles>

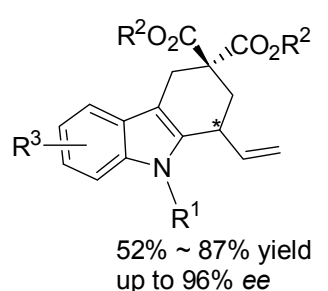<smiles>[Y20]c1cccc(OC)c1-c1c(OC)cccc1P(=O)(O)O</smiles>

$\mathrm{R}^{1}=\mathrm{H}, \mathrm{Me} ; \mathrm{R}^{2}=\mathrm{H}$, alkyl, halide; $\mathrm{R}^{3}=$ alkyl $\mathrm{Ar}=2,5-\mathrm{t}-\mathrm{Bu}_{2}-4-\mathrm{OMe}-\mathrm{C}_{6} \mathrm{H}_{2}$

\section{3 烯丙醇与羰基化合物的烯丙基化反应}

除了芳香族化合物外，在烯丙基烷基化反应中， 1,3-二羰基化合物也是一种高效的亲核取代试剂. 2006 年, $\mathrm{Baba}$ 等 ${ }^{[28]}$ 报道了 1,3 -二羰基化合物和烯丙醇在 $\mathrm{InCl}_{3}$ 催化作用下发生烷基化反应(表 5, Entry 1).

表 5 酸催化 1,3-二羰基化合物和烯丙醇分子间烷基化反应

Table 5 Acid catalyzed intermolecular allylic alkylation of 1,3-dicarbonyl compounds with allylic alcohols
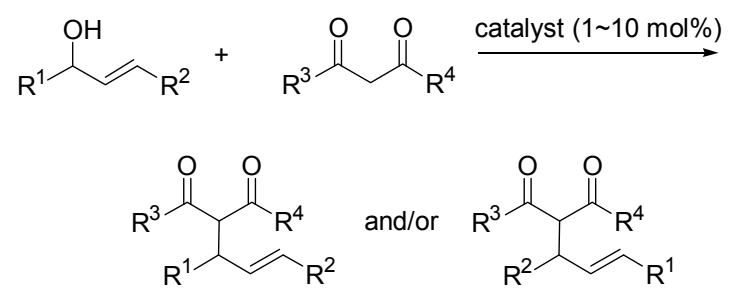

$\mathrm{R}^{1}, \mathrm{R}^{2}=\mathrm{H}$, alkyl, aryl

$\mathrm{R}^{3}, \mathrm{R}^{4}=$ alkyl, aryl, alkoxy

\begin{tabular}{clll}
\hline Entry & \multicolumn{1}{c}{ Catalyst } & Yield/\% & Ref. \\
\hline 1 & $\mathrm{InCl}_{3}$ & $75 \sim 95$ & {$[28]$} \\
2 & $\mathrm{Bi}(\mathrm{OTf})_{3}$ & $62 \sim 73$ & {$[29]$} \\
3 & $\mathrm{Ln}(\mathrm{OTf})_{\mathrm{n}}$ & $51 \sim 92$ & {$[30]$} \\
4 & $\mathrm{Yb}(\mathrm{OTf})_{3}$ & $72 \sim 93$ & {$[31]$} \\
5 & $\mathrm{FeCl}_{3}$ & $72 \sim 82$ & {$[32]$} \\
6 & $\mathrm{I}_{2}$ & $51 \sim 99$ & {$[33]$} \\
7 & $p-\mathrm{TsOH} \bullet \mathrm{H}_{2} \mathrm{O}$ & $80 \sim 72$ & {$[34]$} \\
\hline
\end{tabular}

在温和的反应条件下, 烯丙基烷基化产物的产率为 $79 \%$ \% $\%$. 随后的研究工作表明, 其他 Lewis 酸类催 化剂如 $\mathrm{Bi}(\mathrm{OTf})_{3}{ }^{[29]}, \mathrm{Ln}(\mathrm{OTf})_{n}(\mathrm{Ln}=\mathrm{Yb}, \mathrm{La}, \mathrm{Hf} ; n=3$, $4)^{[30]}, \mathrm{Yb}(\mathrm{OTf})_{3}{ }^{[31]}$ 和 $\mathrm{FeCl}_{3}{ }^{[32]}$ 都是 $\mathrm{C}-\mathrm{C}$ 键形成的高效催 化剂(表 5, Entries 2～5).

Chan 等 ${ }^{[33]}$ 以 $I_{2}$ 为催化剂催化 1,3 -二羰基化合物和 
烯丙醇的烯丙基烷基化反应(表 5, Entry 6), 结果表明当 使用对水和空气都稳定的分子 $I_{2}$ 作为催化剂时, 仍可得 到烯丙基化产物，相应的产率高达 99\%. 另外, Sanz 等 ${ }^{[16]}$ 以聚合物固载 $p-\mathrm{TsOH} \cdot \mathrm{H}_{2} \mathrm{O}$ 为催化剂, 催化 1,3 二 二 羰基化合物和烯丙醇进行烯丙基烷基化 (表 5, Entry 7), 该固体酸催化剂的特点是催化剂可以通过过滤回收, 并 且适用于大规模工业化生产.

2014 年 Ohshima 等 ${ }^{[34]}$ 报道了微波促进的 Pt 和四氢 吡咯作为共催化剂进行烯丙醇的直接烷基化反应, 如 Eq. 4 所示 ${ }^{[35]}$. 该反应的最大特点是得到高选择性的单 烯丙基化 1,3-二羰基化合物，选择性高达 99\%.
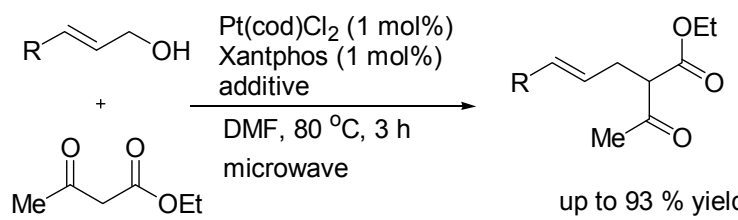

2014 年, Zhang 等 ${ }^{[35]}$ 发表了酮或者醛羰基的 $\alpha$-位烯 丙基烷基化反应, 如 Eq. 5 所示. 该反应使用 Pd 作为催 化剂, 同样需要四氢吡咯, 在常温就可以得到相应产率 高达 99\%的烯丙基化产物, 并且进行了相应的机理研 究.

$$
\text { up to } 99 \% \text { yield }
$$

烯丙醇除了能被羰基的 $\alpha$-位进攻还可以与羰基氧 原子发生作用. 同样是 2014 年, Floreancig 等 ${ }^{[36]}$ 报道了 羰基氧在 $\mathrm{Re}_{2} \mathrm{O}_{7}$ 催化下与烯丙醇反应，接着形成的中间 体被 $\mathrm{Et}_{3} \mathrm{SiH}$ 还原成四氢呋喃杂环衍生物的研究工作 (Scheme 2). 通过改变原料碳链的长度还可以合成氧杂 六元环, 另外除硅烷亲核试剂外, 他们还考察了其它的 亲核试剂, 例如烯丙基硅烷、炔基硼烷、烯醇硅醚等, 相 应的产物都取得了不错的产率.

\section{4 烯丙醇分子间胺基化反应}

Sanz 等 ${ }^{[16]}$ 以聚合物固载 $p-\mathrm{TsOH} \cdot \mathrm{H}_{2} \mathrm{O}$ 为催化剂, 催 化烯丙醇和对硝基苯胺进行胺基化反应, 得到的胺基化 产物得率为 $86 \%$ (表 6, Entry 1). 之后, Shibasaki 等 ${ }^{[37]}$ 对 烯丙基直接胺基化反应进行了深入研究, 在 $\mathrm{Bi}(\mathrm{OTf})_{3}$ 催 化作用下, 烯丙醇分别与磺酰胺、氨基甲酸酯以及酰胺 进行反应 (表 6, Entry 2). 研究结果表明, 当加入 $\mathrm{KPF}_{6}$ 作

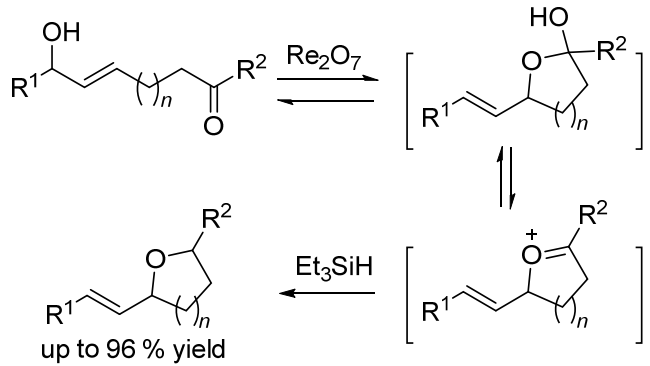

图式 $2 \mathrm{Re}_{2} \mathrm{O}_{7}$ 催化的杂环合成

$\begin{array}{lll}\text { Scheme } 2 & \operatorname{Re}_{2} \mathrm{O}_{7} \text { catalyzed heterocycle synthesis }\end{array}$

表 6 酸催化烯丙基醇分子间烯丙基胺基化反应

Table 6 Acid catalyzed intermolecular allylic amination with allylic alcohols

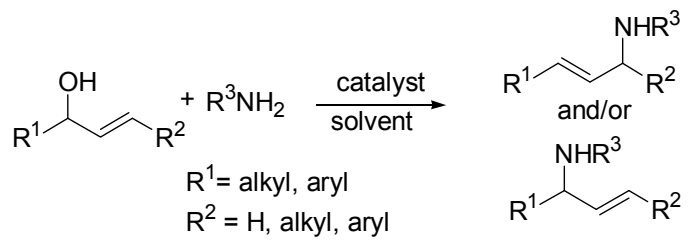

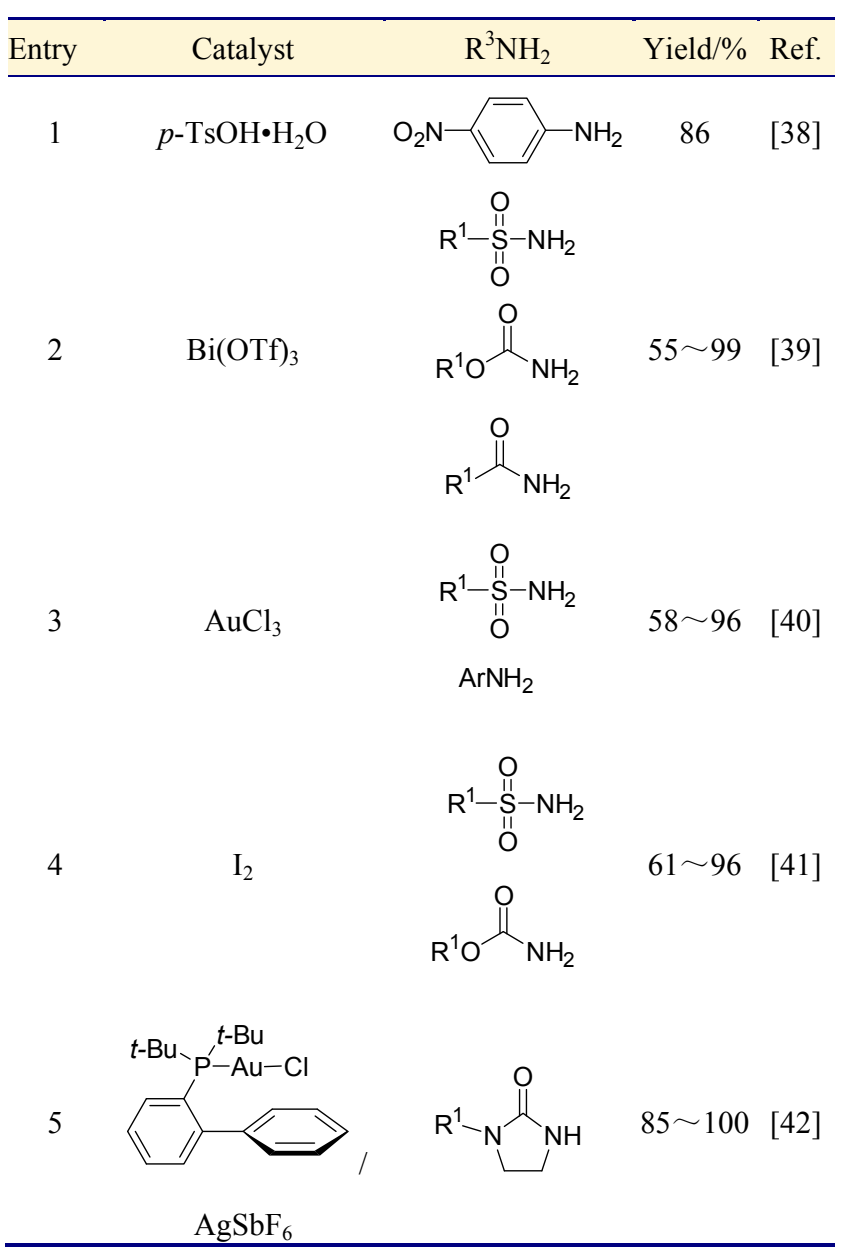

为助催化剂时，能够有效地促进该反应的进行，相应的 胺类产物产率为 $55 \% \sim 99 \%$. 与此同时, Liu 等 ${ }^{[38]}$ 研究发 现，在分子间烯丙基胺基化反应中，当使用 $\mathrm{AuCl}_{3}$ 为催 化剂时, 除了磺酰胺外, 取代苯胺也是高效的亲核试剂 
(表 6, Entry 3). 在室温条件下, 相应的胺基化产物产率 在 $58 \% \sim 96 \%$. 1 年后, Chan 小组 ${ }^{[39]}$ 报道了在室温条件 下, 以分子 $\mathrm{I}_{2}$ 为烯丙醇和磺酰胺或氨基甲酸酯胺基化反 应的催化剂, 取得了良好的催化效果(表 6, Entry 4). 最 近, Widenhoefer 等 ${ }^{[40]}$ 研究了烯丙醇和五元环艮在金催 化剂的作用下，进行烯丙醇的胺基化反应. 结果表明, 当以 $\left[\mathrm{P}(t-\mathrm{Bu})_{2}(o\right.$-biphenyl $\left.)\right] \mathrm{AuCl}$ 和 $\mathrm{AgSbF}_{6}$ 物质的量的比 $1: 1$ 原位产生金催化剂时, 分子间的胺基化产物具有 较高的空间位置选择性，相应的产物的产率在 $85 \%$ 100\%(表 6, Entry 5).

2008 年, Liang 等 ${ }^{[41]}$ 报道了以 $\mathrm{HAuCl}_{4} \cdot \mathrm{H}_{2} \mathrm{O}$ 为催化 剂, 催化环状烯丙基炔丙醇和对甲基苯磺酰胺进行分子 间烯丙基胺基化反应，接着再分子内胺基化反应，最后 得到高取代的吡咯衍生物(Scheme 3). 反应分两步胺基 化进行, 首先生成烯丙基胺中间产物, 随后进一步分子 内烯丙基胺关环反应生成多取代的吡咯衍生物, 相应的 产物产率高达 $83 \%$. 该反应有两大缺点，一是该反应只 适用于环己醇衍生物进行胺基化反应, 且胺的用量为醇 用量的 15 倍，此外还需要大量的金催化剂(20 mol\%).

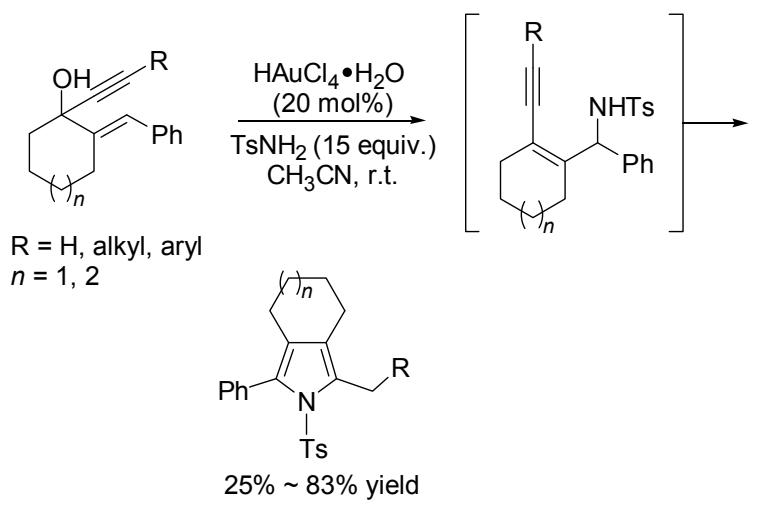

图式 3 金催化烯丙基胺基化/氢胺化串联反应

Scheme 3 Gold catalyzed tandem allylic amination/hydroamination

Liu 等 ${ }^{[42]}$ 采用相似的方法合成吡咯衍生物, 在 $\mathrm{Au} / \mathrm{Ag}$ 复合催化条件下, 烯炔醇和对硝基苯胺或对甲基 苯磺酰胺进行胺基化/分子内氢胺化反应(Scheme 4). 研 究表明, 在室温条件下 $\left(p-\mathrm{MeOC}_{6} \mathrm{H}_{4}\right)_{3} \mathrm{PAuCl}$ 和银盐催化 烯丙醇胺基化反应也是通过两步法进行的, 首先烯丙醇 在催化剂作用下生成中间产物烯丙基胺, 然后进一步分 子内胺基环化得到 40\% 48\%产率的相应的吡咯衍生 物.

2014 年, Peters 等 ${ }^{[43]}$ 报道了 Pd 催化的烯丙醇与异氰 分子间的反应, 以二茂铁衍生物作为手性配体, 经过烯 丙醇羟基与异氧的加成反应, 然后再分子内重排得到手 性的烯丙基磺胺衍生物, 相应的产率高达 $90 \%$, ee 值高 达 98\% (Eq. 6).

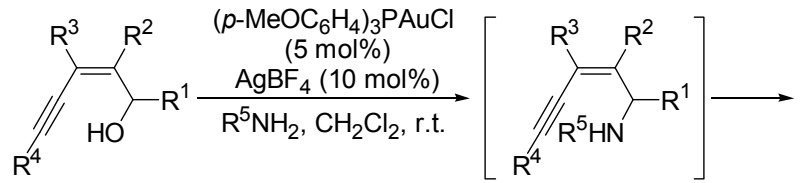

$\mathrm{R}^{1}-\mathrm{R}^{4}=\mathrm{H}$, alkyl, aryl

$\mathrm{R}^{5}=\mathrm{Ts}$ or $p-\mathrm{NO}_{2} \mathrm{C}_{6} \mathrm{H}_{4}$

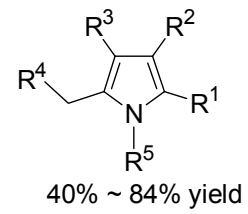

图式 4 金催化烯丙胺基化/氢胺化串联反应

Scheme 4 Gold catalyzed tandem allylic amination/hydroamination

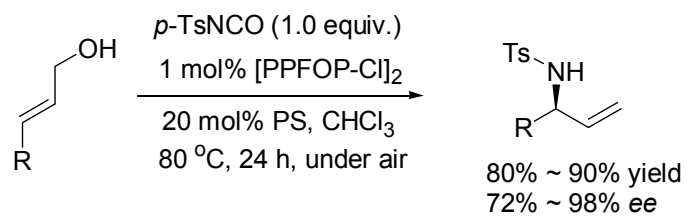

2014 年, Chan 课题组 ${ }^{[44]}$ 也发表了分子间的烯丙基 胺基化合成吡咯衍生物的反应(Eq. 7). 我们利用烯丙基 炔丙基二醇这样特殊的底物，与芳基磺酰胺首先分子间 烯丙基酰胺化反应，然后再分子内磺胺与炔丙基醇进行 分子内的酰胺化反应得到高达 94\%产率的多取代吡咯 衍生物. 为了验证磺酰胺首先与烯丙醇发生反应, 我们 通过低温降低反应的活性，成功地合成出了烯丙基酰胺 化的中间体，间接说明了反应发生的区域选择性. 但是 该反应也有缺点，亲核试剂只能是芳基磺酰胺，脂肪族 磺酰胺不适用于本反应.

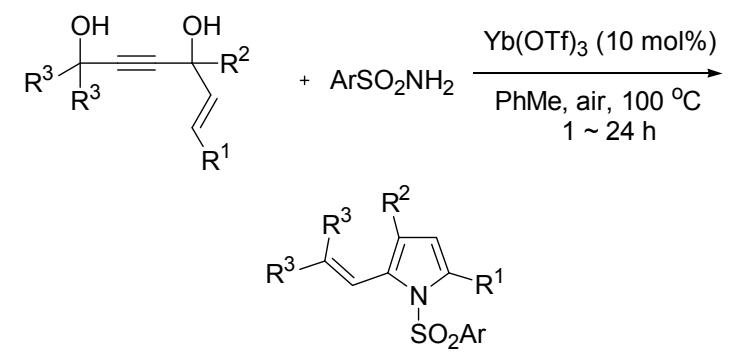

up to $94 \%$ yield

\section{5 烯丙醇分子内胺基化或酰胺化反应}

2008 年, Nishizawa 等 ${ }^{[45]}$ 报道了用 $\mathrm{Hg}(\mathrm{OTf})_{2}$ 催化取 代烯丙醇在室温条件下进行分子内胺基化反应. 相应的 含氮杂环化合物的产率为 $41 \% \sim 99 \%$. 两年后，同样是 该反应，只是 Yamamoto 等 ${ }^{[48]}$ 发现将手性膦配体 $(R)$-BINAPHANE 和 $\mathrm{Hg}(\mathrm{OTf})_{2}$ 形成的配合物作为手性催 化剂, 可以获得相应的手性含氮杂环产物(Eq. 8). 研究 表明该催化剂的突出优点在于具有高效的对映选择性, 当催化剂用量为 $1 \%$ 时, 相应的手性产物的产率在 91\% 99\%, ee 值高达 99\%. 

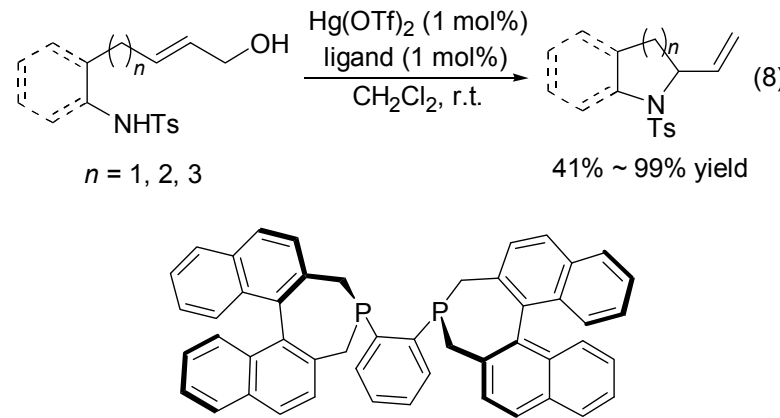

ligand: $(R)$-BINAPHANE

2010 年, Cossy 等 ${ }^{[47]}$ 以 $\mathrm{FeCl}_{3} \cdot 6 \mathrm{H}_{2} \mathrm{O}$ 为催化剂, 催化 烯丙醇分子内进行六元胺化环合反应, 生成顺式 2,6-哌 啶(Eq. 9). 研究表明, 顺式异构体热稳定性较好, 在室 温下反应条件下的得到相应的产率 $51 \% \sim 100 \%$.

$$
\begin{array}{ll}
\mathrm{R}^{3} \mathrm{O}^{2} \\
\mathrm{X}=\mathrm{NPG}, \mathrm{O} & \mathrm{R} \mathrm{CH}_{2} \mathrm{Cl}_{2} \text {, r.t. } \\
\mathrm{R}^{1}, \mathrm{R}^{2}=\mathrm{H} \text {, alkyl, aryl } & \\
\mathrm{R}^{3}=\mathrm{H}, \mathrm{Ac} &
\end{array}
$$

最近, Widenhoefer 等 ${ }^{[48]}$ 报道了烯丙醇分子内 1,3 -手 性转换的胺基化合成方法(Eq. 10). 研究表明, 当使用 $\left[\mathrm{P}(t-\mathrm{Bu})_{2} o\right.$-biphenyl $] \mathrm{AuCl}$ 和 $\mathrm{AgSbF}_{6}$ 以 $1: 1$ 比例混合作 为催化剂时, 烯丙醇分子内胺基化产物哌啶的产率高达 99\%, ee 值高达 96\%.<smiles>CC(O)/C=C\CCCCc1ccccc1</smiles>

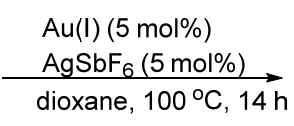

$(R, Z)$

$96 \%$ ee

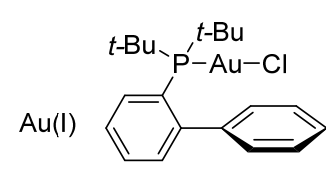

与此同时, Kawai 等 ${ }^{[49]}$ 报道了将烯丙醇进行手性胺 基化反应, 得到了手性胺基化产物四氢异喹啉衍生物 (Eq. 11). 研究表明, 在 $\mathrm{Bi}(\mathrm{OTf})_{3}$ 催化过程中, 出现 1,3手性转变, 得到的产物具有良好的空间选择性以及较好 的产率, 另外研究还表明, 产物的立体化学受到 $\mathrm{S}_{\mathrm{N}} 2^{\prime}$ 反 应过程的控制, 因此苯环上的取代基对产物的立体化学 具有重要的影响.

2009 年, Chan 等 ${ }^{[50]}$ 报道了金复合催化剂催化磺酰 胺烯丙醇进行 6-endo-trig 胺基化反应(Scheme 5). 当以 甲苯为溶剂, 在催化剂 $\mathrm{AuCl}_{3}$ 和 $\mathrm{AgSbF}_{6}$ 存在的条件下, 室温反应即可得到六元环化产物, 相应的产率 $40 \%$

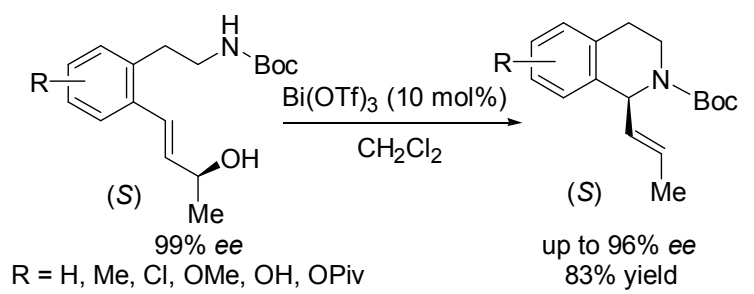

$91 \%$. 此外, 该合成方法的实用性是可以用来合成具有 生物活性的天然产物 $( \pm)$-angustureine.

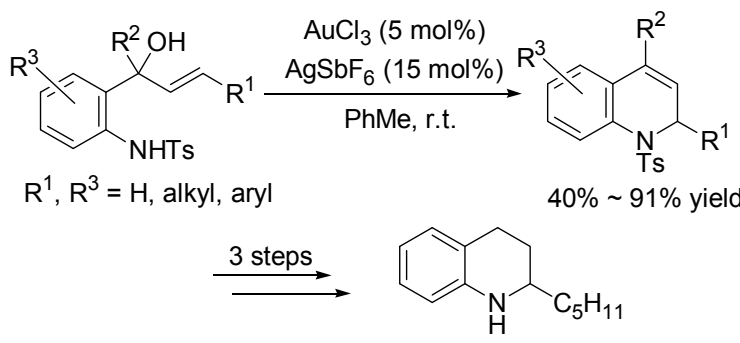

( \pm )angustureine

图式 5 金催化烯丙醇分内胺基化反应

Scheme 5 Gold catalyzed intramolecular amination of allyl alcohol $^{[52]}$

\section{6 烯丙醇烷氧基化反应}

单烯丙基二醇既作为亲核试剂又作为亲电试剂是 由 Aponick等 ${ }^{[51]}$ 首先提出来的(Eq. 12). 单烯丙基二醇在 催化剂 $\mathrm{Ph}_{3} \mathrm{PAuCl} / \mathrm{AgOTf}$ 催化作用下, 生成顺式四氢吡 喃或顺式四氢呋喃. 当反应温度为 $-78{ }^{\circ} \mathrm{C}$, 催化剂用 量为 $0.1 \%$ 时, 相应产物产率为 $79 \% \sim 99 \%$, 且 $d r>25$ ： 1.

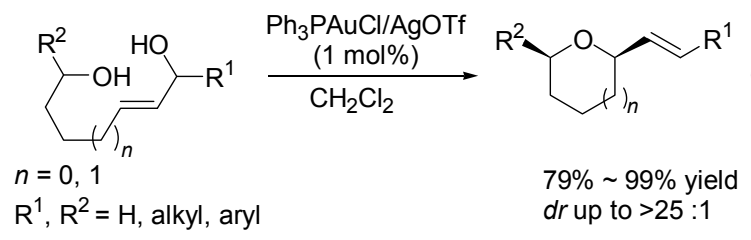

两年后该课题组设计了一种烯丙醇酚类化合物，经 过 6-endo-trig 分子内烷氧化反应, 得到了相应的黄酮衍 生物 (Eq. 13 $)^{[52]}$. 该反应是以 $\mathrm{THF}$ 为溶剂, $\left[\mathrm{P}(t-\mathrm{Bu})_{2}-\right.$ (o-biphenyl)] $\mathrm{AuCl} / \mathrm{AgOTf}$ 为催化剂, 产物的收率高达 $91 \%$.

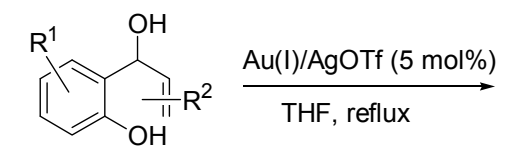

$\mathrm{R}^{1}, \mathrm{R}^{2}=\mathrm{H}$, alkyl, aryl

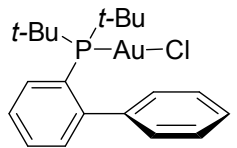

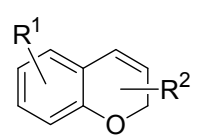


紧接着 2010 年, Bandini 等 ${ }^{[53]}$ 利用金催化剂催化手 性烯丙醇原料, 通过 $\mathrm{Au}_{2} \mathrm{Cl}_{2}(\mathrm{dppf})$ 和 $\mathrm{AgNTf}_{2}$ 原位产生金 催化剂, 进行分子内烷氧基化反应, 得到手性的氮氧杂 六元环化合物(Eq. 14). 该反应需在低温下反应, 相应产 物的产率为 $41 \% \sim 94 \%$ 之间, ee 值高达 95\%.

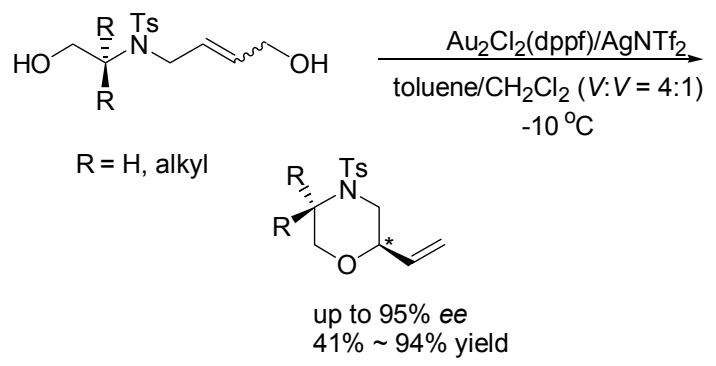

2011 年, Aponick 等 ${ }^{[54]}$ 使用手性单烯丙基二醇在 $\mathrm{Ph}_{3} \mathrm{PAuCl} / \mathrm{AgOTf}$ 催化作用下, 发生 1,3-手性转移生成 手性四氢吡喃衍生物(Eq. 15), 该研究表明, 反应原料手 性单丙烯基二醇的烯烃的几何构型对产物的立体化学 影响很大.

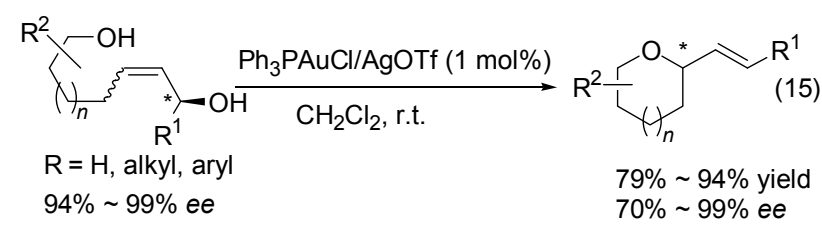

最近, Rueping 等 ${ }^{[55]}$ 报道了烯丙醇在强酸性手性布 朗斯特酸催化作用下, 进行对映选择性分子内烷氧基化 反应(Eq. 16). 在低温条件下, 相应手性产物苯并吡喃的 产率在 $61 \% \sim 95 \%, e e$ 值高达 $96 \%$. 另外, 该文提出该反 应的对映选择性诱导模式涉及到一个由手性布朗特酸 与烯丙醇原料生成的手性离子对, 该手性离子对对产物 的立体构型产生了影响.<smiles>[R]C=CC([R])(O)c1ccccc1O</smiles>

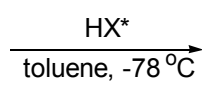

$\mathrm{R}^{1}=\mathrm{H}$, alkyl

$\mathrm{R}^{2}=$ alkyl $\mathrm{R}^{3}=$ aryl<smiles>O=P(F)(F)N[P+]([O-])(Oc1c(-c2ccccc2)cc2c(c1-c1c(-c3ccccc3)cc3c(c1-c1ccccc1)CCCC3)CCCC2)OC(F)(F)F</smiles>

烯丙醇分子间烷氧基化反应也有报道, 主要是发生 分子间的醚化反应. 例如早在 1997 年, Namura 等 ${ }^{[58]}$ 报 道了烯丙醇与酚在 $\mathrm{Pd}(\mathrm{OAc})_{2} / \mathrm{PPh}_{3}$ 以及 $\mathrm{Ti}(\mathrm{OPr}-i)_{4}$ 共催化 剂作用的条件下进行醚化反应研究, 获得了高达 $89 \%$
的烯丙醇醚化产物, 而且没有检测到酚的邻位以及对位 进攻的产物，在该催化反应条件下，酚的进攻具有较高 的选择性(Eq. 17).

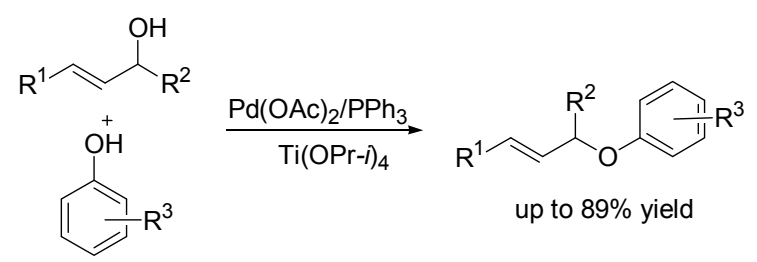

烯丙基醇与脂肪醇的分子间反应主要是进行醚化 反应. 如 2008 年, Chan 等 ${ }^{[57]}$ 在验证亲核试剂与烯丙醇 反应机理的时候进行了烯丙醇的双分子醚化反应研究. 在分子碘催化下，烯丙醇发生两分子自身醚化反应，在 较短的时间内获得了 $97 \%$ 的醚化产物(Eq. 18).
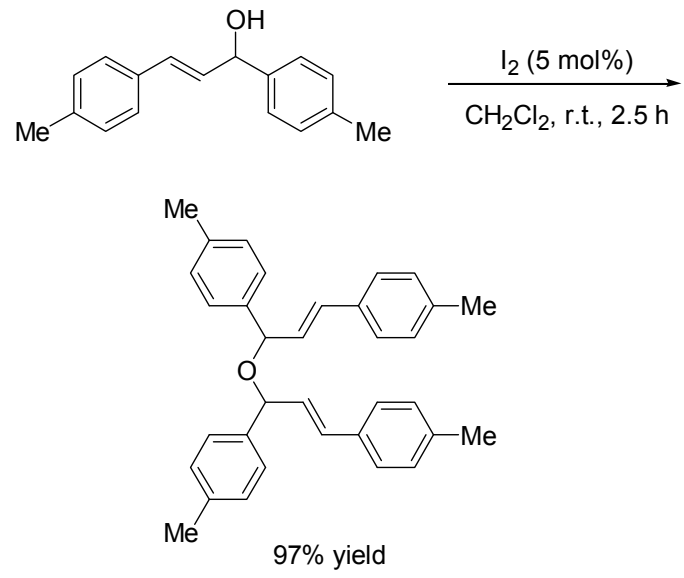

\section{2 结论与展望}

综上所述, 在现代有机合成化学中, 以 Lewis 酸和 Brønsted 酸催化亲电试剂前体烯丙醇, 进行亲核取代烯 丙基化反应构筑 $\mathrm{C}-\mathrm{X}(\mathrm{X}=\mathrm{C}, \mathrm{N}, \mathrm{O}, \mathrm{S})$ 键的合成方法, 具有原材料烯丙醇来源广泛或者容易制备、水是其唯一 的副产物、合成方法通用性较强、催化剂对环境友好等 优点. 尽管这一新方法具有众多的优点, 但是以 Lewis 酸和 Brønsted 酸催化进行烯丙基化反应的研究还处于 起步阶段, 大多数的烯丙基化产物是消旋化合物, 而手 性烯丙基化的研究还严重不足, 高对映选择性手性烯丙 基化反应的研究将成为烯丙基化理论研究的重要发展 方向.

随着烯丙基化研究的不断深入，必将面临众多的挑 战，因此以下几个方面应值得注意：(1)烯丙醇原料结构 还是比较单一、简单的, 有必要设计出结构新颖的烯丙 醇原料，进行烯丙基化反应合成出特殊结构的化合物; (2)烯丙基化催化剂大多使用的是均相催化剂, 大多数 是不可再生循环的, 有必要开发出催化活性高、易与产 
物分离、且能循环使用的非均相高效催化剂如固体酸催 化剂; (3)因手性烯丙基化反应的研究刚起步, 有必要开 展立体化学的手性控制基础理论研究, 例如从手性催化 剂、配体以及手性原料等几个方面开展对产物立体化学 的影响研究.

\section{References}

[1] Tsuji, J. Transition Metal Reagents and Catalysts, Wiley, New York, 2000.

[2] (a) Tsuji, J. Acc. Chem. Res. 1969, 2, 144.

(b) Trost, B. M. Tetrahedron 1977, 33, 2615.

(c) Trost, B. M. Acc. Chem. Res. 1980, 13, 385.

(d) Trost, B. M.; Verhoeven, T. R. Comprehensioe Organometallic Chemistry; Pergamon: Oxford, 1982, pp. 799 938.

(e) Trost, B. M. J. Organomet. Chem. 1986, 300, 263.

[3] For reviews on allylation, see: (a) Yus, M.; González-Gómez, J. C.; Foubelo, F. Chem. Rev. 2013, 113, 5595.

(b) Mejuch, T.; Gilboa, N.; Gayon, E.; Wang, H.; Houk, K. N.; Marek, I. Acc. Chem. Res. 2013, 46, 1659.

(c) Yus, M.; González-Gómez, J. C.; Foubelo, F. Chem. Rev. 2011, 111,7774 .

(d) Tietze, L. F.; Kinzel, T.; Brazel, C. C. Acc. Chem. Res. 2009, 42,367 .

For selected examples on allylation, see: (e) Tao, Z.-L.; Li, X.-H.; Han, Z.-Y.; Gong, L.-Z. J. Am. Chem. Soc. 2015, 137, 4054.

(f) Deng, H.-P.; Wang, D.; Szabó, K. J. J. Org. Chem. 2015, 80, 3343.

(g) Feng, T.; Si, C.; Liu, R.; Fan, X.; Wei, B. Chin. J. Org. Chem. 2013, 33, 1291 (in Chinese).

(冯涛, 司长梅, 刘如成, 范翔, 魏邦国, 有机化学, 2013, 33, 1291.)

(h) Min, Q.-Q.; Yin, Z.; Feng, Z.; Guo, W.-H.; Zhang, X. J. Am. Chem. Soc. 2014, 136, 1230.

(i) Chen, W.; Chen, M.; Hartwig, J. F. J. Am. Chem. Soc. 2014, 136, 15825 .

(j) Sheffy, F. K.; Godschalx, J. P.; Stille, J. K. J. Am. Chem. Soc. 1984, 106, 4833.

(k) Godschalx, J. P.; Stille, J. K. Tetrahedron Lett. 1980, 21, 2599.

(1) Moreno-Manas, M.; Pajuelo, F.; Pleixats, R. J. Org. Chem. 1995, 60, 2396.

(m) Miyaura, N.; Yamada, K.; Suginome, H.; Suzuki, A. J. Am. Chem. Soc. 1985, 107, 972.

(n) Evans, P. A.; Nelson, J. D. J. Am. Chem. Soc. 1998, 120, 5581.

(o) Okude, Y.; Hirano, S.; Hiyama, T.; Nozaki, H. J. Am. Chem. Soc. 1977, 99, 3179.

[4] Trost, B. M. Acc. Chem. Res. 2002, 35, 695.

[5] Bandini, M.; Tragni, M. Org. Biomol. Chem. 2009, 7, 1501.

[6] Constable, D. J. C.; Dunn, P. J.; Hayler, J. D.; Humphrey, G. R.; Leazer, J. L., Jr.; Linderman, R. J.; Lorenz, K.; Manley, J.; Pearlman, B. A.; Wells, A.; Zaks, A.; Zhang, T. Y. Green Chem. 2007, $9,411$.

[7] Tsuji, J.; Takahashi, H.; Morikawa, M. Tetrahedron Lett. 1965, 6, 4387.

[8] Tsuchimoto, T.; Tobita, K.; Hiyama,T.; Fukuzawa, S. J. Org. Chem. 1997, 62, 6997.

[9] Piao, C. R.; Zhao, Y. L.; Han, X. D.; Liu, Q. J. Org. Chem. 2008, 73, 2264.

[10] Zhang, X.; Rao, W.; Sally; Chan, P. W. H. Org. Biomol. Chem. 2009, 7, 4186.
[11] Rao, W.; Chan, P. W. H. Org. Biomol. Chem. 2008, 6, 2426.

[12] Chen, Y.; Lu, Y.; Li, G.; Liu, Y. Org. Lett. 2009, 11, 3838.

[13] McCubbin, J. A.; Hosseini, H.; Krokhin, O. V. J. Org. Chem. 2010, 75,959

[14] Niggemann, M.; Meel, M. J. Angew. Chem., Int. Ed. 2010, 49, 3684.

[15] Davies, H. J. Appl. Chem. 1959, 9, 137.

[16] Sanz, R.; Martínez, A.; Miguel, D.; Álvarez-Gutiérrez, J. M.; Rodríguez, F. Adv. Synth. Catal. 2006, 348, 1841.

[17] Bras, J. L.; Muzart, J. Tetrahedron 2007, 63, 7942.

[18] Liu, Y. L.; Liu, L.; Wang, Y. L.; Han, Y. C. Wang, D.; Chen, Y. J. Green Chem. 2008, 10, 635.

[19] Yasuda, M.; Somyo, T.; Baba, A. Angew. Chem., Int. Ed. 2006, 45, 793.

[20] Yadav, J. S.; Subba Reddy, B. V.; Aravind, S.; Narayana Kumar, G. G. K. S.; Reddy, A. S. Tetrahedron Lett. 2007, 48, 6117.

[21] Jana, U.; Maiti, S.; Biswas, S. Tetrahedron Lett. 2007, 48, 7160.

[22] Zhou, X.; Zhang, H.; Xie, X.; Li, Y. J. Org. Chem. 2008, 73, 3958.

[23] Guo, S.; Liu, Y. Org. Biomol. Chem. 2008, 6, 2064.

[24] Wang, J.; Zhang, L.; Jing, Y.; Huang, W.; Zhou, X. Tetrahedron Lett. 2009, 50, 4978.

[25] Namba, K.; Yamamoto, H.; Sasaki, I.; Mori, K.; Imagawa, H.; Nishizawa, M. Org. Lett. 2008, 10, 1767.

[26] Bandini, M.; Eichholzer, A.; Kotrusz, P.; Tragni, M.; Troisi, S.; Umani-Ronchi, A. Adv. Synth. Catal. 2009, 351, 319.

[27] Bandini, M.; Eichholzer, A. Angew. Chem., Int. Ed. 2009, 48, 9533.

[28] Yasuda, M.; Somyo, T.; Baba, A. Angew. Chem., Int. Ed. 2006, 45, 793.

[29] Rueping, M.; Nachtsheim, B. J.; Kuenkel, A. Org. Lett. 2007, 9, 825.

[30] Noji, M.; Konno, Y.; Ishii, K. J. Org. Chem. 2007, 72, 5161.

[31] Huang, W.; Wang, J.; Shen, Q.; Zhou, X. Tetrahedron Lett. 2007, $48,3969$.

[32] Jana, U.; Biswas, S.; Maiti, S. Tetrahedron Lett. 2007, 48, 4065.

[33] Rao, W.; Tay, A. H. L.; Goh, P. J.; Choy, J. M. L.; Ke, J. K.; Chan, P. W. H. Tetrahedron Lett. 2008, 49, 122.

[34] Shibuya, R.; Lin, L.; Nakahara, Y.; Mashima, K.; Ohshima, T. Angew. Chem., Int. Ed. 2014, 53, 4377.

[35] Huo, X.; Yang, G.; Liu, D.; Liu, Y.; Gridnev, I. D.; Zhang, W. Angew. Chem., Int. Ed. 2014, 53, 6776.

[36] Xie, Y.; Floreancig, P. E. Angew. Chem., Int. Ed. 2014, 53, 4926.

[37] Qin, H.; Yamagiwa, N.; Matsunaga, S.; Shibasaki, M. Angew. Chem., Int. Ed. 2007, 119, 413.

[38] Guo, S.; Song, F.; Liu, Y. Synlett 2007, 964.

[39] Wu, W.; Rao, W.; Er, Y. Q.; Loh, J. K.; Poh, C.Y.; Chan, P. W. H. Tetrahedron Lett. 2008, 49, 2620.

[40] Mukherjee, P.; Widenhoefer, R. A. Org. Lett. 2010, 12, 1184.

[41] Shu, X.; Liu, X.; Xiao, H.; Ji, K.; Guo, L.; Liang, Y. Adv. Synth. Catal. 2008, 350, 243.

[42] Lu, Y.; Fu, X.; Chen, H.; Du, X.; Jia, X.; Liu, Y. Adv. Synth. Catal. 2009, 351, 129.

[43] Bauer, J. M.; Frey, W.; Peters, R. Angew. Chem., Int. Ed., 2014, 53, 7634.

[44] Zhang, X.; Teo, J. W.; Ma, D. L.; Leung, C. H.; Chan, P. W. H. Tetrahedron Lett. 2014, 55, 6703.

[45] Namba, K.; Nakagawa. Y.; Yamamoto, H.; Imagawa, H.; Nishizawa, M. Synlett 2008, 1719.

[46] Yamamoto, H.; Ho, E.; Namba, K.; Imagawa, H.; Nishizawa, M. Chem. -Eur. J. 2010, 16, 11271.

[47] Guérinot, A.; Serra-Muns, A.; Gnamm, C.; Bensoussan, C.; Reymond, S.; Cossy, J. Org. Lett. 2010, 12, 1808.

[48] Mukherjee, P.; Widenhoefer, R. A. Org. Lett. 2011, 13, 1334. 
[49] Kawai, N.; Abe, R.; Matsuda, M.; Uenishi, J. J. Org. Chem. 2011, 76, 2102.

[50] Kothandaraman, P.; Foo, S. J.; Chan, P. W. H. J. Org. Chem. 2009, $74,5947$.

[51] Aponick, A.; Li, C.-Y.; Biannic, B. Org. Lett. 2008, 10, 669.

[52] Aponick, A.; Biannic, B.; Jong, M. R. Chem. Commun. 2010, 46, 6849.

[53] Bandini, M.; Monari, M.; Romaniello, A.; Tragni, M.
Chem. -Eur. J. 2010, 16, 14272.

[54] Aponick, A.; Biannic, B. Org. Lett. 2011, 13, 1330.

[55] Rueping, M.; Uria, U.; Lin, M. Y.; Atodiresei, I. J. Am. Chem. Soc. 2011, 133, 3732.

[56] Satoh, T.; Ikeda, M.; Miura, M.; Nomura, M. J. Org. Chem. 1997, $62,4877$.

[57] Rao, W.; Tay, A. H. L.; Goh, P.J.; Choy, J. M. L.; Ke, J. K.; Chan, P. W. H. Tetrahedron Lett. 2008, 49, 112.

(Qin, X.) 